\title{
Correlated X-ray and optical variability in KV UMa
}

\author{
H. C. Spruit ${ }^{1}$ and G. Kanbach ${ }^{2}$ \\ 1 Max-Planck-Institut für Astrophysik, Postfach 1317, 85741 Garching bei München, Germany \\ 2 Max-Planck-Institut für Extraterrestrische Physik, Postfach 1312, 85741 Garching bei München, Germany
}

Received 28 March 2002 / Accepted 21 May 2002

\begin{abstract}
We present coordinated X-ray and optical observations of the X-ray transient KV UMa (=XTE J1118+480) at high time resolution. The optical variation associated with spikes in the X-rays consist of a dip preceding the spike by $0-5 \mathrm{~s}$, and a peak following it. The cross-correlation function between X-rays and optical has the same shape. It rises sharply, within $30 \mathrm{~ms}$ of the peak of the X-rays. The shape of the cross correlation shows significant intrinsic variability on time scales as short as $30 \mathrm{~s}$. Analyzing this variability with principal component analysis (calibrated with simulated data), we find two statistically independent components. The first is similar to the average cross-correlation. The second also consists of a dip followed by a peak, but on a $3 x$ shorter time scale. These properties of the optical light, together with the high optical brightness of the transient, are not easily explained by reprocessing of the X-rays.
\end{abstract}

Key words. accretion: accretion disks - black hole physics - X-rays

\section{Introduction}

The transient X-ray source XTE J1118+480 (=KV UMa) appeared in January 2000 (Remillard et al. 2000) and (with an interruption in February) was active as an X-ray source till end of July. The source appears to be a relatively nearby $(2 \mathrm{kpc})$ binary with an orbital period of $4 \mathrm{hrs}$, and a K-star companion. Its mass function of $6 M_{\odot}$ makes the primary a solid black hole candidate, (Mc Clintock et al. 2001a; Wagner et al. 2001). The X-ray outburst was somewhat unusual compared with "standard" transients such as X-ray Nova Muscae. The $\mathrm{X}$-ray flux was relatively constant during the main outburst episode (March-July). The X-ray luminosity (1-100 keV) was only about $10^{36} \mathrm{erg} / \mathrm{s}$, and the source was always found in a $\mathrm{X}$-ray hard state (most of the X-ray energy flux appearing around $100 \mathrm{keV}$ ). Since hard state spectra are the rule during the decay phase of $\mathrm{X}$-ray transients, this suggests that the accretion rate of the system was significantly below the Eddington value.

Due to the low galactic absorption at the position of the source, its spectral energy distribution (SED) could be determined from the IR to X-rays, including the crucial EUV part of the spectrum (Hynes et al. 2000; McClintock et al. 2000b), a unique result for X-ray transients. These observations showed that the source had an unusually high optical-to-X-ray flux ratio compared with other transients. The SED appears to peak at $100 \mathrm{eV}$, and the inferred UV-to-EUV flux of $10^{36} \mathrm{erg} / \mathrm{s}$ was about the same as the X-ray flux. The optical-to-X-ray flux

Send offprint requests to: $\mathrm{H}$. C. Spruit, e-mail: henk@mpa-garching.mpg.de ratio was about 0.1 . High optical brightness during a hard $\mathrm{X}$-ray state has been seen before in the unusual long-term variable source GX 339-4 (Motch et al. 1983).

The long duration of this transient, its location above the galactic plane, and its relatively close distance made it an ideal target for simultaneous X-ray and optical observations. Though such coordinated observations have been attempted regularly, successful observations at high (sub-second) time resolution have been reported previously in only two cases. Motch et al. (1981) reported correlated fast optical and X-ray variability from 96 s of observations of GX 339-4, when it was in one of its rapidly variable and optically bright states. The X-ray-optical cross-correlation of these data was reported to show a negative correlation preceding the X-rays by a few seconds. Hynes et al. (2000) reported simultaneous HST and XTE observations of the black hole transient GRO J1655-40. The cross-correlation showed, in contrast with the case of 339-4, a positive correlation peak following the X-rays, interpreted by the authors as evidence of reprocessing of $\mathrm{X}$-rays by the accretion disk.

The optical emission of black hole candidates in outburst thus appears puzzling, both in its unpredictable amplitude, and its contradictory correlations with the X-rays. Theoretically, the optical light is interesting because it may provide a way to probe the structure of the accretion flow, if the light is caused by reprocessing of X-rays from the central object (e.g. Arons \& King 1993). Evidence for such reprocessing of X-rays by an accretion disk has been found in type-1 X-ray bursts from accreting neutron stars (Matsuoka et al. 1984; Turner et al. 1985; van Paradijs et al. 1990; Kong et al. 2000). 
On the other hand, lower limits to the optical brightness temperatures inferred from the short optical variability in GX 339-4 led Fabian et al. (1982) to conclude that a more likely mechanism for the optical radiation in the Motch et al. data is thermal cyclosynchrotron emission in a strong magnetic field. We reached a similar conclusion in a first description and interpretation of the results reported here (Kanbach et al. 2001). If this interpretation is correct, the regions contributing most to the optical-UV light would be much closer to the black hole than in a reprocessing model, at least in objects like KV UMa and GX 339-4, and optical light of black hole transients would potentially be a powerful new diagnostic of the inner accretion flow.

In Kanbach et al. (2001) we found that the average $\mathrm{X}$-ray/optical cross correlation function consists of a peak starting at zero lag, preceded by a "precognition dip". It thus combines both of the effects seen previously by Motch et al. and Hynes et al. This raises the question how these two components of the cross correlation are related, in particular since the dip at negative lag is not very easily explained in models proposed so far. In this paper we address this question by a more detailed statistical analysis of our observations of KV UMa. The theoretical interpretation will be developed further in a future paper.

\section{Observations}

X-ray and optical observations were collected on the nights of 4, 5, 6 and 7 July 2001. X-ray observations were made by the Rossi X-ray Timing Explorer, for about one orbit on each of these nights. The total duration of useful simultaneous observations was 2.5 hours. The optical observations were done with the OPTIMA photometer (Straubmeier et al. 2001) attached to the $1.3 \mathrm{~m}$ telescope on Mt. Skinakas, Crete (http://observatory.physics.uoc.gr/), which is operated jointly by the Foundation for Research and Technology Hellas (FORTH) and the Max-Planck-Institut für Extraterrestrische Physik. The photometer consists of a cluster of fiber-coupled avalanche diodes. The diodes are sensitive from 450 to $950 \mathrm{~nm}$ at a mean quantum efficiency of $50 \%$. Individual photon arrival times are recorded to an accuracy of $2 \mu \mathrm{s}$ with a GPS-based clock, at a maximum count rate of $200000 / s$. On several of the nights, the telescope was facing into a fairly strong wind, causing modulations of the signal at frequencies around 9 and $40 \mathrm{~Hz}$ due to telescope vibration. Most of the results reported here are based on the correlation between optical and X-ray signals, for which this artefact has little consequence. In the cross-correlation functions it appears as a source of noise but has no systematic effects.

The average total X-ray count rate was $500 \mathrm{~s}^{-1}$ (3 detectors), typical for the brightness of the source during most of its outburst. The optical brightness was around $V=13$, resulting in a count rate of about $24000 \mathrm{~s}^{-1}$. While the X-ray flux was highly variable, with amplitudes typical of the hard states of black hole transients or Cyg X-1, the rms variability of the optical flux was only about $10 \%$ on time scales of $100 \mathrm{~ms}-30 \mathrm{~s}$.
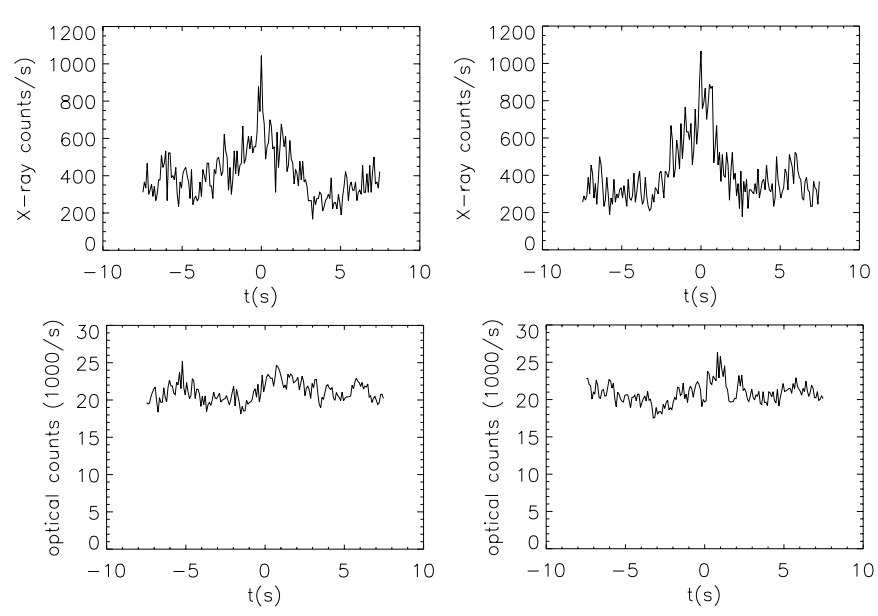

Fig. 1. Two examples of the X-ray and optical light curves, around the time of peaks in the X-rays.
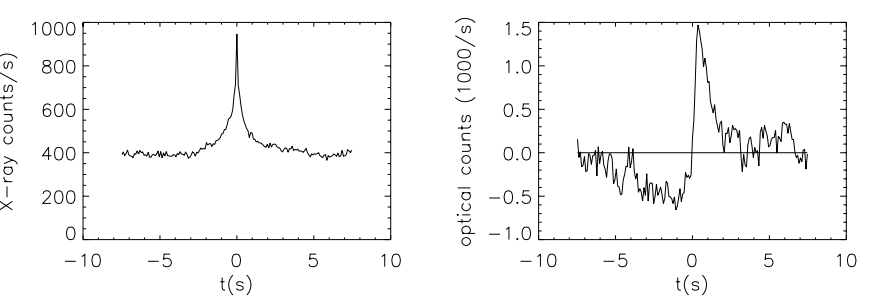

Fig. 2. Average X-ray and optical light curves of 100 peak-aligned events like the ones shown in Fig. 1.

\section{Analysis}

An example of the X-ray and optical light curves is shown in Fig. 1. The data show the original count rates, without background or baseline subtractions. Peaks in the X-rays with durations of a few seconds as seen in this example were fairly typical. Visual inspection of such events suggests that the corresponding optical signal consists of a dip, preceding the $\mathrm{X}$-rays by several seconds, followed by a positive response peaking a few seconds after the X-rays.

In order to quantify this impression in a more objective way, an automatic peak-finding algorithm was devised to collect and superimpose these events. The X-ray time series was compared with a box-car smoothed version of itself (with a width of $30 \mathrm{~s}$ ). Time intervals where the actual signal was more than twice the smoothed signal were identified as peaks. The 100 events found in this way were aligned in time on the maxima of the X-ray peaks.

The average X-ray and optical light curves of the peak events found in this way is shown in Fig. 2. This average confirms the subjective impression given by Fig. 1. The dip in the optical preceding the $\mathrm{X}$-rays appears to be a systematic effect, though its amplitude varies between events.

\subsection{Cross-correlation}

The superposition of events shown in Fig. 2 is limited in its time resolution by the width of the X-ray peaks, of the order of a second. A view at much higher time resolution, at the expense of a somewhat less direct interpretation, is given by the 

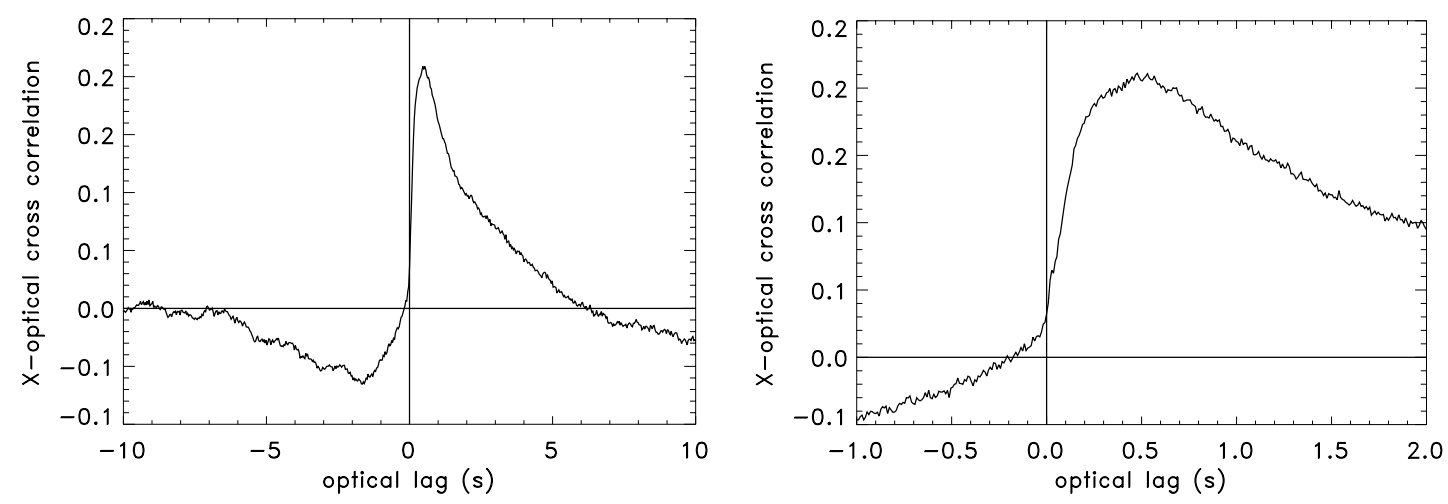

Fig. 3. Average X-ray/optical cross correlation from 297 sample of the time series. Right panel shows the cross correlation on an expanded scale; note the sharp rise at zero lag.

cross correlation between the data. The cross-correlation of the $\mathrm{X}$-ray time series $S_{\mathrm{X}}$ and the optical series $S_{\mathrm{O}}, C_{\mathrm{XO}}(t)=$ $S_{\mathrm{X}} * S_{\mathrm{O}}$, where a $*$ denotes the convolution, was computed for 297 independent segments of data of $25 \mathrm{~s}$ length. The average of these cross-correlations is shown in Fig. 3.

The cross correlation contains a residual of the photon noise in the original time series. In Figs. 3, 4 this shows up as the weak high-frequency noise component. Its amplitude is much lower, however, than the noise due to the intrinsic variability of the cross correlation, as Fig. 4 shows.

The cross-correlation appears to contain significant signal for lags (optical later than X-rays) from about -5 to $+5 \mathrm{~s}$. On these time scales, the correlation shows the same properties as the direct comparison of the signals shown in Fig. 2: a dip followed by a peak of about the same amplitude.

As the right panel of Fig. 3 shows, the change from the dip to the peak takes place quite close to zero lag. The slope of the correlation increases by a factor 10 within about $30 \mathrm{~ms}$ at $t=0$, and $50 \%$ of the peak is reached at $t=100 \mathrm{~ms}$.

\subsection{Dip and peak}

A peak starting at $t=0$ is understandable in a reprocessing model for the optical light, and its duration is also of the order expected for reprocessing of X-rays by an accretion disk. Evidence for such reprocessing has been seen in X-ray bursters (Matsuoka et al. 1984; van Paradijs et al. 1990; Kong et al. 2000). It has also been advanced to explain the optical response in some black hole transients (O'Brien \& Horne 2000; Hynes et al. 1998). A delayed optical response is also a natural result in the outflow model of Kanbach et al. (2001). In this model, the unusually large optical flux of KV UMa does not come from the accretion disk but instead is produced by synchrotron emission in a magnetic outflow. The dip in the optical light preceding the X-rays is not very natural in either of these explanations, however. This raises the question whether the dip should be regarded as an independent phenomenon, or as a part of the correlation function associated directly with the peak.

The question whether the dip represents an independent physical process from that producing the peak can be answered to some extent by investigating the variability of the dip and the peak between different samples of the cross correlation.
If their amplitudes turn out to be statistically uncorrelated, it is unlikely that they are due to the same process. Significant variability is in fact present in both the dip and the peak. This is shown in Fig. 4, which presents a random subset of 16 of the 300 correlations. From this figure it is also clear, however, that a large part of the variability of the cross correlation appears to be independent of both the dip and the peak. In many cases, this additional component masks the dip and/or the peak. From simple inspection of figures like these, it is hard to determine if this additional variability is just some form of intrinsic random variability in the source, or whether it contains additional systematic variations.

\section{Principal components of the cross correlation}

A more quantitative way of investigating the variability of a signal like the cross correlations in Fig. 4 is by a principal component analysis (PCA, e.g. Kendall 1980, not to be confused here with the PCA detectors on board of XTE). If the signal is variable, and a sufficiently large set of samples of it available, this method can decompose the samples into components whose amplitudes vary statistically independently through the set. The method has been used in astronomy, among other applications, for the classification of galaxy spectra from large surveys (e.g. Folkes et al. 1999), AGN spectra (Boroson 2002), Chepheid light curves (Kanbur et al. 2002), and velocity distributions in molecular clouds (Brunt \& Kerton 2002). The components found in this way can often be interpreted more easily in terms of variations in physically quantities than the original (large) datasets.

In practice, significant care has to be excercised in interpretating the principal components. In the following we do this by comparison with forward modeling. First we apply PCA to our set of 297 samples of the cross correlation and interpret a few of the most significant components. We then verify the stability and significance of these components by constructing sets of synthetic cross correlation functions, and processing them in the same way. These synthetic samples consist of superpositions of a few input components whose shapes are guessed, with statistical properties similar to those of the real set of cross correlations. The input components and the statistics of their amplitudes are adjusted to find the minimal number of "true" 

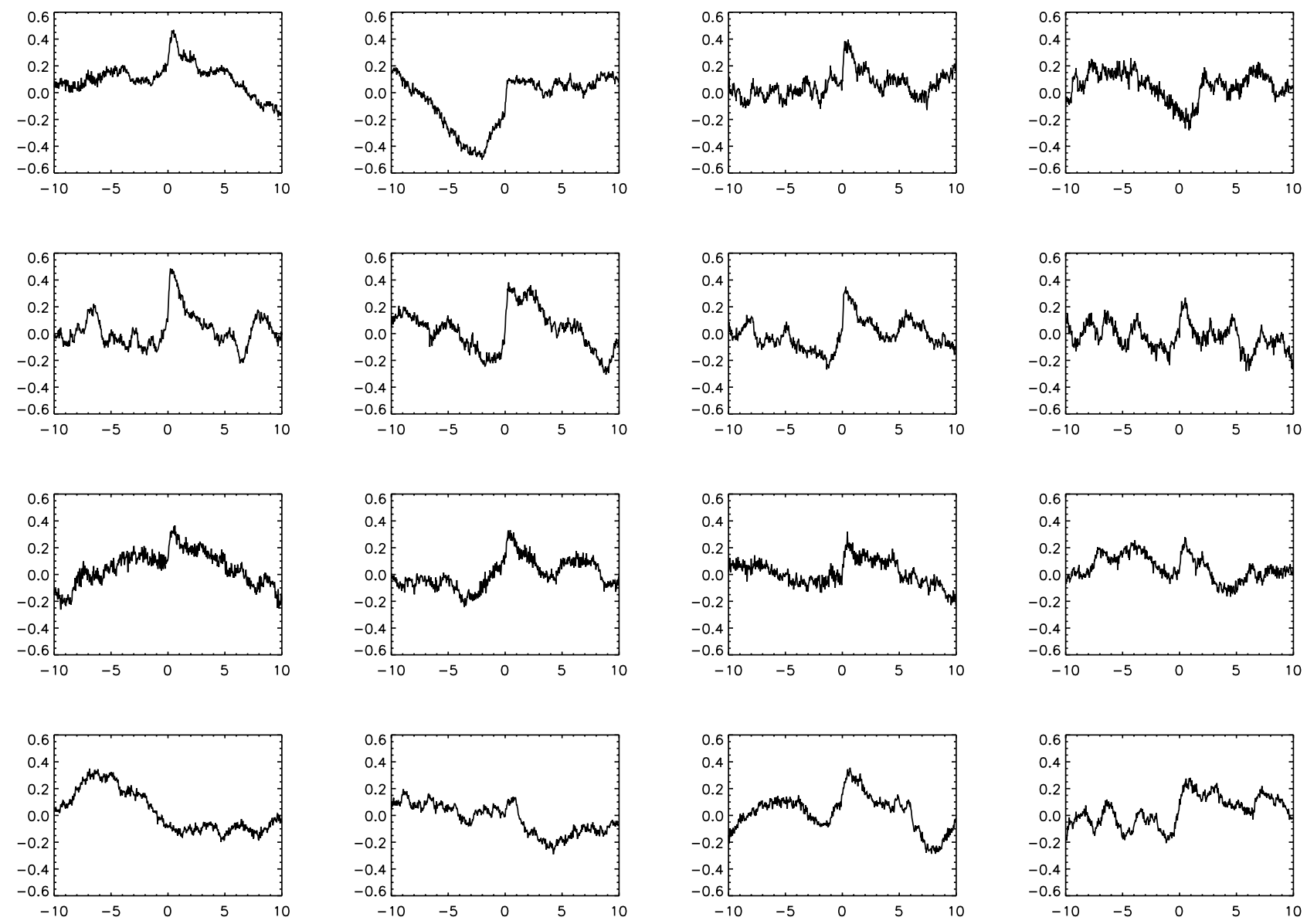

Fig. 4. 16 randomly selected samples from the set of 297 X-ray/optical cross correlation functions.

components, and their shapes, that are needed to reproduce the pattern of components found by the PCA of the real data set.

\subsection{Method}

For our application, the PCA is used as follows. Let the $\mathrm{X}-\mathrm{O}$ cross correlation function of sample $j$ be denoted by $\mathbf{a}^{j}(t)$. Let $m$ be the number of available samples of the correlation function ( $m=297$ in the present application), discretize the timedelay axis $t$ in $n$ bins $t_{i}\left(-12.5<t_{i}<12.5 \mathrm{~s}, n=150\right.$ in our case), and write $a_{i}^{j}=\mathbf{a}^{j}\left(t_{i}\right)$. We treat the $n$ time bins as the variables whose statistical properties are to be found by PCA. Since neighboring time bins are highly correlated, the number of degrees of freedom is much less than $n$. The degree of correlation of the time bins with each other contains information on the actual number of degrees of freedom in the signal. We measure the correlation between time bins $i$ and $k$ by the covariance matrix $C_{i k}$ :

$C_{i k}=\sum_{j}\left(a_{i}^{j}-\bar{a}_{i}\right)\left(a_{k}^{j}-\bar{a}_{k}\right)$,

where $\overline{\mathbf{a}}=\sum_{j} \mathbf{a}^{j} / m$ is the average of $\mathbf{a}$ in the set. The principal components of $\mathbf{a}$ are then conventionally defined as those linear combinations of the columns of $a_{i}^{j}$ that diagonalize the covariance matrix. (In many PCA applications, the correlation matrix is used instead of the covariance matrix. This is important if the variables measured are different physical quantities. For our case, this makes practically no difference, since the variables $a_{i}$ are the discretized values of a single continuous function.) If the coefficients of these linear combinations are denoted by the matrix $c_{i k}$, the principal components $p_{k}$ are given by

$p_{k}^{j}=\sum_{i} c_{k i} a_{i}^{j}$

and the samples themselves are given, in terms of the principal components, by the inverse of $c$,

$a_{i}^{j}=\sum_{k} c_{i k}^{-1} p_{k}^{j}$

These components are somewhat abstract, since a linear combination of different time bins is not a physically very meaningful quantity in our application. A more useful representation of the principal components is found by noting that the quantity

$a_{k i}^{j}=c_{i k}^{-1} p_{k}^{j}$

describes the amplitude of principal component $k$ in the $i$ th time bin of the $j$ th sample. The average of this amplitude over the set of samples,

$P_{k i} \equiv \sum_{j} a_{k i}^{j} / m$

describes the principal components in the "time domain", i.e. as a decomposition of our average cross correlation function. 
We use this representation for the rest of our analysis. The average of the cross correlation function over the set, $\overline{\mathbf{a}}$, is the sum of these principal components:

$\bar{a}_{i}=\sum_{k} P_{k i}$

\subsection{Results}

The covariance matrix is singular if the number of time bins $n$ is larger than the number of samples $m$. This limits the time resolution that can be used for the discretization of our cross correlation functions to 300 points ${ }^{1}$. The results depend somewhat on the range in $t$ used for the analysis. Most of the signal of interest appears between $t= \pm 5 \mathrm{~s}$. Using this range, and bins of $30 \mathrm{~ms}$ yields the principal components shown in Fig. 5 .

The amplitude $A$ of components varies both in absolute value and sign. For the first component we find that the average is larger than the variation, but for the higher components the average is generally small compared to the variation. Instead of showing the components at their average amplitude $\bar{A}$, we have therefore plotted them at a scale that takes into account both the average and the variation. The value used for this, somewhat arbitrarily, is $\tilde{A}=\left(\bar{A}^{2}+\sigma_{A}^{2}\right)^{1 / 2}$, where $\sigma_{A}$ is the rms deviation of $A$.

The first two components together account for $67 \%$ of the variance between the samples of the cross correlation function, if a time-lag interval $(-\Delta t<t<\Delta t)$ with $\Delta t=5 \mathrm{~s}$ is used. The first component appears quite similar to the average cross correlation. The second component peaks around the "precognition dip", though it has the opposite sign and extends somewhat to positive lag as well. If the time interval used is shorter, the first two components account for a larger fraction of the variation, and less for a longer interval. For $\Delta t=2 \mathrm{~s}$ the first two components account for $86 \%$ of the variance, for $\Delta t=12 \mathrm{~s}$ only $44 \%$. This is understandable if the cross correlation consists of a signal of relatively stable shape concentrated around $t=0$, plus an unsystematically varying component that is spread out over a wider range in the time delay coordinate $t$.

The nature of the remaining components is not obvious at this point. Some of them, such as $P_{3}$ and $P_{5}$, appear to contain signals related to the sharp rise of the cross correlation at $t=0$. The PCA also interprets pure noise in the data in terms of additional components, and it is not a priori obvious which components are systematic and which may be just artefacts. A useful test to see which of the components is stable with respect to noise is to divide the set of samples in two halves and comparing the components derived from each of these subsets. This is shown in Fig. 6. The four curves shown result from two different ways of dividing the set in halves. In the first, the two subsets consist of the even numbered and the odd numbered samples of the (chronologically ordered) set. The difference

1 This limitation can be circumvented by data-compression of the samples. A convenient compression is obtained by applying a wavelettransform to the samples. PCA is then applied on the first $n_{0} \leq m$ wavelet coefficients, and the resulting principal components transformed back to the time domain by the inverse wavelet transform. For $n_{0}=n$ this process is loss-free and yields identical results.
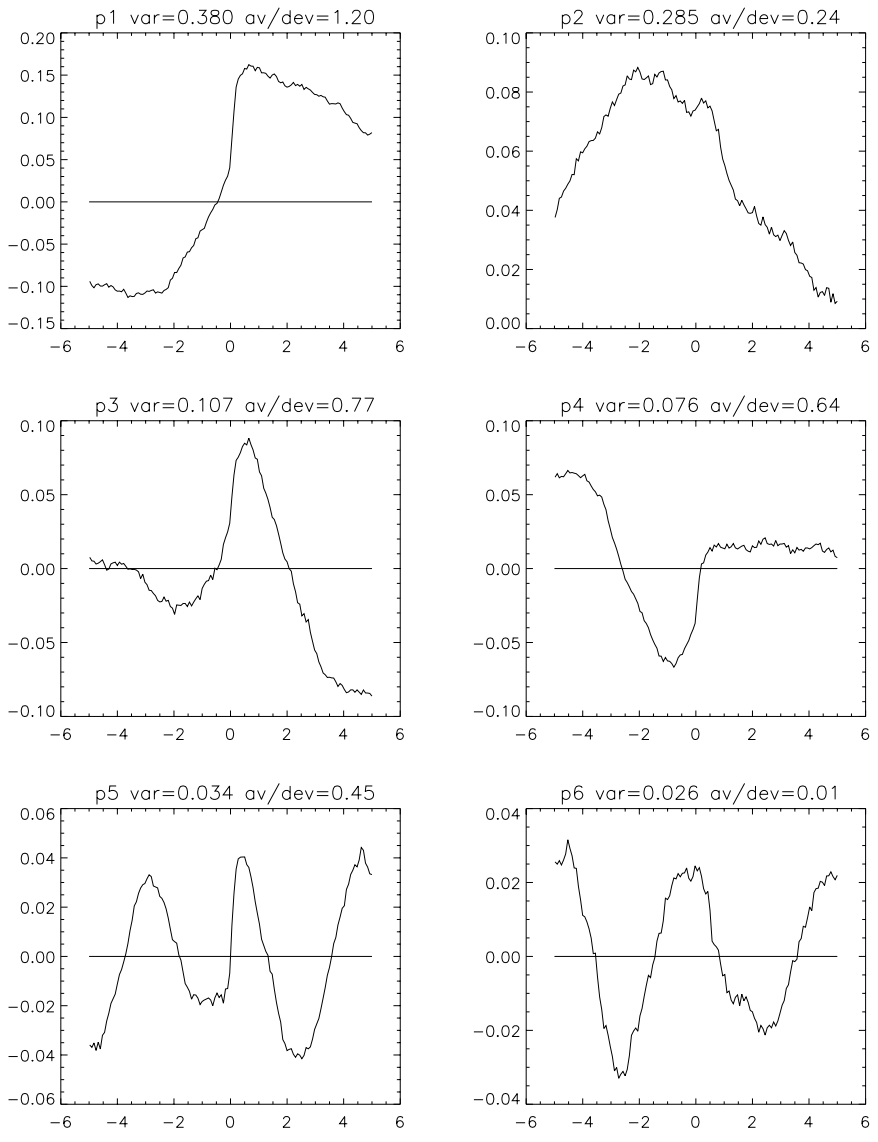

Fig. 5. The first six principal components from a PCA of the set of 297 samples of the X-ray/optical cross correlation. Numbers above the panels show the fraction of the variance in the cross correlation accounted for by the components, followed by ratio of the average to the deviation of the component's amplitude.

between these two subsets measures the intrinsic variability of the cross correlation on a time scale of $25 \mathrm{~s}$ (the duration of a sample). In the second division, the first half of the set is compared with the second half. This measures the variability on time scales of days.

As Fig. 6 shows, the components, especially the higher ones, are remarkably stable on both the short and the long time scales. From this one would be tempted to conclude that all six components shown are real. The process by which the components are derived in PCA, however, leaves the possibility open that some of the components are artefacts. This is because the components are constructed as orthogonal directions in the abstract $n$-dimensional space of variables. The directions of insignificant higher components (constructed out of noise) are thus biased in a systematic way by the directions of the significant lower components.

The only way to test which of the components is real in this sense is by comparison of the results with a synthetic data set.

\subsubsection{Tests with synthetic data}

To test the significance of the components, we have constructed synthetic data and applied PCA to these data in the same way as was done with the real data. By varying the input data, 

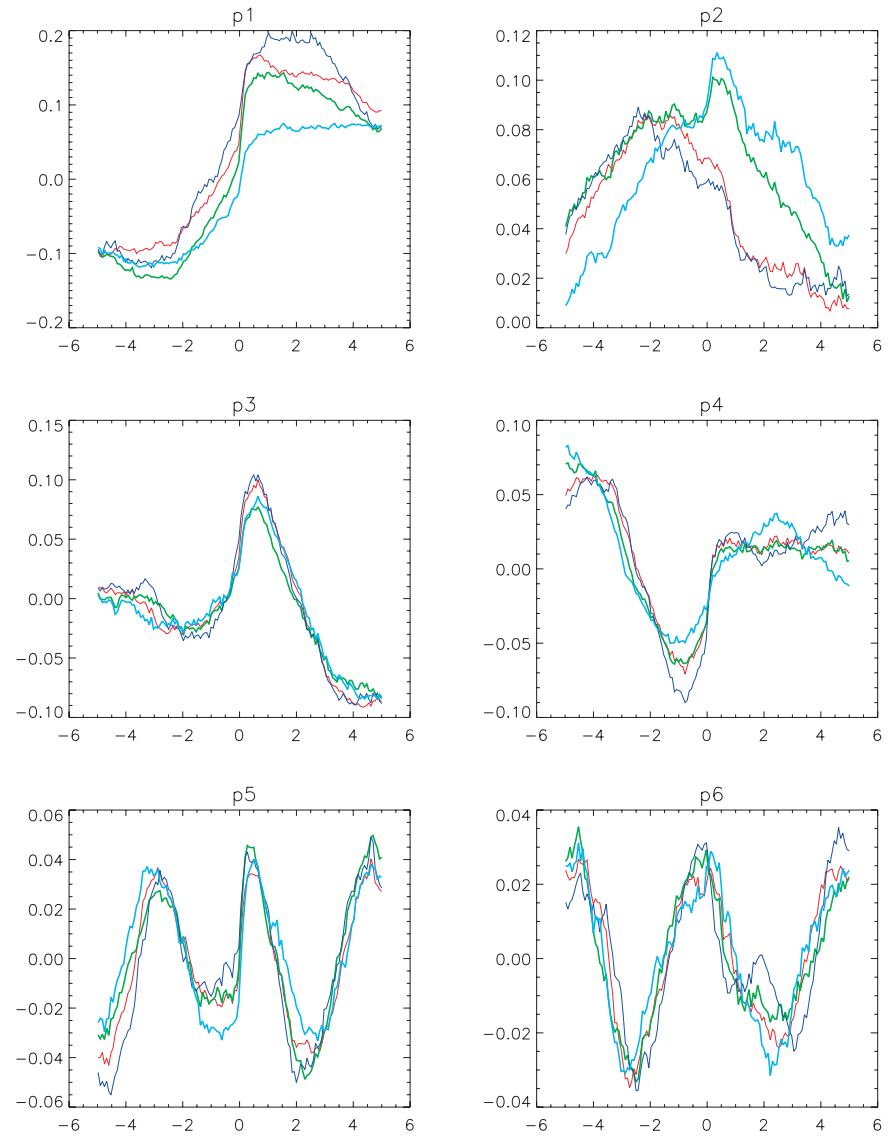

Fig. 6. Stability of the pincipal components with respect to time variability of the data. The full set of correlation functions has been divided into halves in two ways. The dark lines show the even and odd numbered samples (chronologically ordered), measuring differences on a time scale of $25 \mathrm{~s}$. The lighter lines show the first and second halves of the set, indicating differences on a time scale of hours to days. The higher components appear to be quite stable in this sense, but in fact most of these turn out to be artefacts (see text and Fig. 8).

the resulting PCs can be adjusted for agreement. Since many of the components found are suspected to be spurious, the aim of the excercise was to find the minimum number of independently varying signals needed to reproduce the components found by PCA of the real data. It is not neccessary to start the synthetic process with time series of the count rates, since the questions to be answered concern the behavior of principal component analysis as applied to our crosscorrelation functions. Thus, the synthetic data constructed consist of samples of the cross correlation function. Each of the samples consists of a linear combination of a number of adjustable functions of the time delay coordinate $t$, here called "signals". The shape of these signals is the same in all samples, but their amplitudes differ. In addition to these signals, we add a random noise component that is different in each sample.

The amplitudes of the signals are assigned randomly between the samples, normally distributed with specified means and deviations. The noise component consists of white noise, low-pass filtered by boxcar averaging with a width $\delta t$. The parameters of the synthetic data are thus: the shapes of the signals,
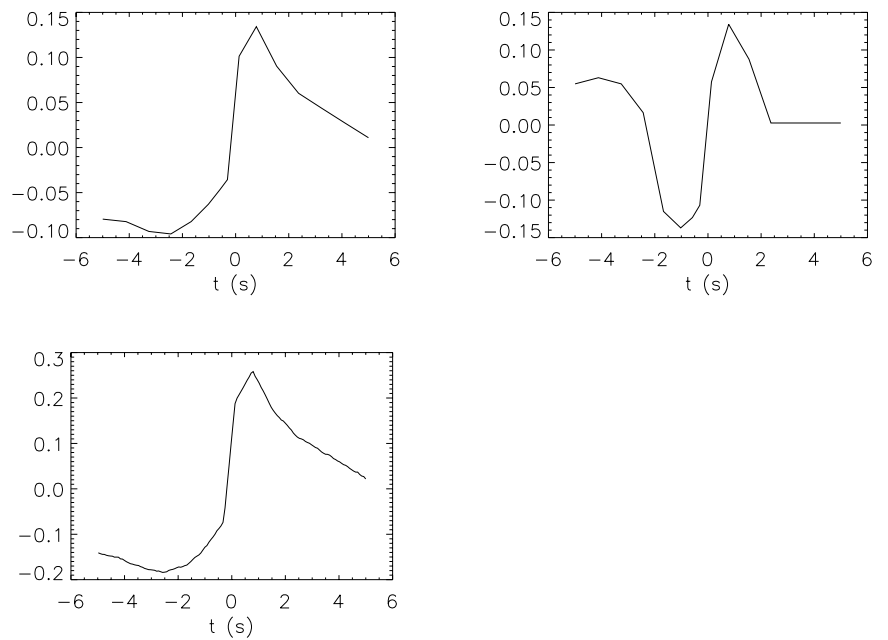

Fig. 7. Top: input components for the synthetic set of cross correlations. Their amplitudes are varied independently. In addition there is a noise component (not shown) whose contribution to the variance peaks around $0.2 \mathrm{~Hz}$. The average cross correlation produced by these components is shown in the bottom panel.

the means $A_{1}$ and $A_{2}$ and deviations $\sigma_{1}, \sigma_{2}$ of their amplitudes, the amplitude $A_{\mathrm{n}}$ of the noise component, and $\delta t$.

PCA on a set of 297 of these synthetic cross correlations yields the components shown in Fig. 8, for a case with two input signals plus a noise component. The shapes of the two input signals were adjusted to fit the shapes of the output components to those of the real data (Fig. 5). The final result for these input signals is shown in Fig. 7

The amplitude of first two PCs of the simulated data are related directly to the average amplitudes of the two input signals. The amplitudes of PCs 3 and higher, however, turn out to be proportional to the amplitude of the boxcar-filtered noise component instead, showing that they are artefacts of the PCA. PCA somehow constructs these higher components out of the noise component. The shapes of these higher components, however, depend sensitively on the shape of the two input signals. The input signals can be determined rather accurately by the fitting process. Two signals plus a noise component turned out to be the minimum number needed. Tests with only one signal plus noise have also been done. These are able to reproduce the first two PCA components, but leave most of the detail in the higher components unexplained.

Except for the first, none of the components found by the PCA resembles the input signals very well. It is thus clear that PCA without comparison with a simulation using synthetic data can not be used to identify the statistically independent components reliably in the present problem. Unfortunately, the forward modeling process was quite cumbersome, owing to the large number of parameters to be adjusted, and is not easily automated.

From this test we conclude that just two independent signal components are needed to explain the observed variability of the cross correlation, apart from an aditional unsystematic noise component. The interpretation of these components presents some puzzles. One might have hoped that the actual independent signals would be the "spike" and the "precognition 

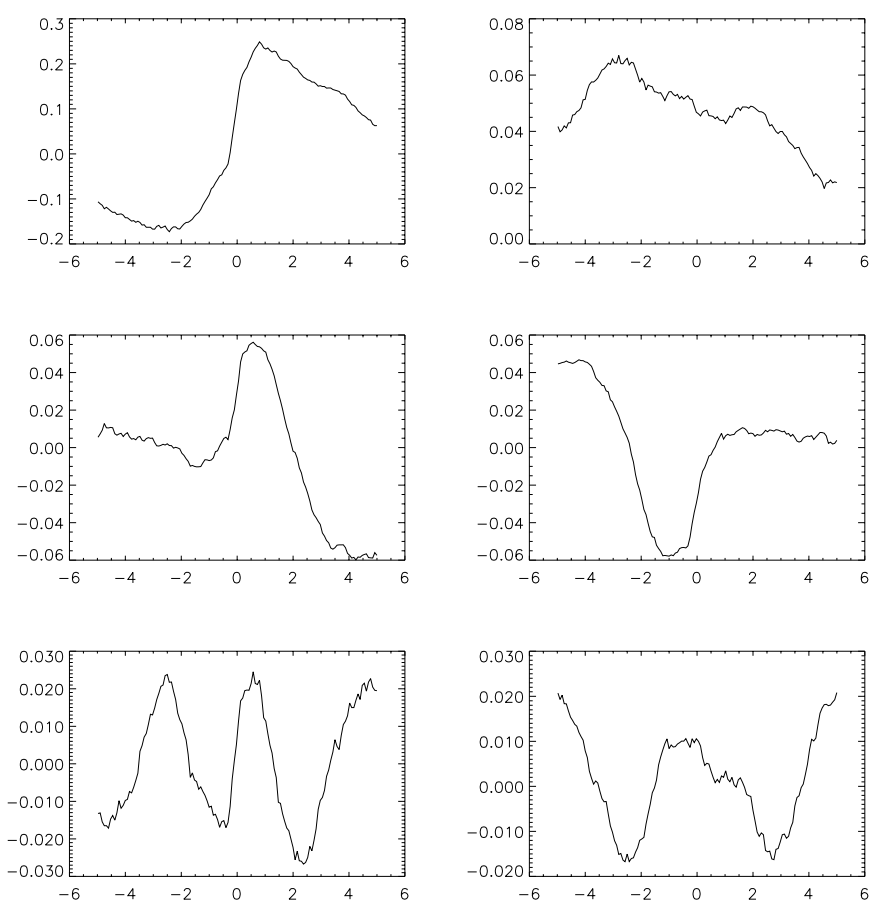

Fig. 8. Components found by PCA of a sample of 297 synthetic cross correlations, constructed from the two input components shown in Fig. 7. The shapes of the input components and their amplitudes were adjusted for agreement of the resulting synthetic PCA components with the first 6 components of the actual data (Fig. 5).

dip", but this turned out not to be the case. Extensive attempts were made to reproduce the PCA components with input signals resembling a pure dip before $t=0$ and a spike after $t=0$, but no satisfactory fit was found. When the shape of the signals was relaxed away from this prejudice, iteration converged onto the signals shown in Fig. 7. Their shapes could be determined rather accurately by this fitting process.

Both signal components consist of a dip followed by a peak. The second signal component resembles a compressed (in time) version of the first, but with the peak being somewhat less pronounced relative to the dip. A possible interpretation is thus that the intrinsic shape of the optical response is a combination of a dip followed by a sharp peak, while the time scale of this pattern varies, by a factor of 3 or so.

\section{Power spectra}

Information complementary to the cross correlations is given by the power spectra. The average X-ray and optical power spectra are shown in Fig. 9. The broad peak near $0.1 \mathrm{~Hz}$ in the $\mathrm{X}$-ray power spectrum is associated with a QPO around this frequency. This QPO has been found in most of the X-ray data from KV UMa (Revnivtsev et al. 2000, Wood et al. 2000), and with high amplitude also in the optical light curve (Haswell et al. 2000; Pavlenko et al. 2001). In our data its amplitude was significantly smaller than in these earlier observations, and undetectable in the optical. Its frequency varied a bit during our observations, with some preference for frequencies of 0.08 and $0.2 \mathrm{~Hz}$.
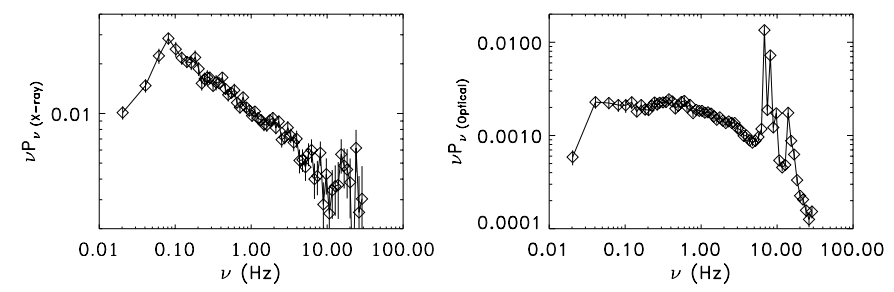

Fig. 9. Average power spectra of the X-ray and optical time series (unit on $y$-axis: variance per factor $e$ in frequency). The peaks in the optical spectrum around $10 \mathrm{~Hz}$ are artefacts due to telescope vibrations.
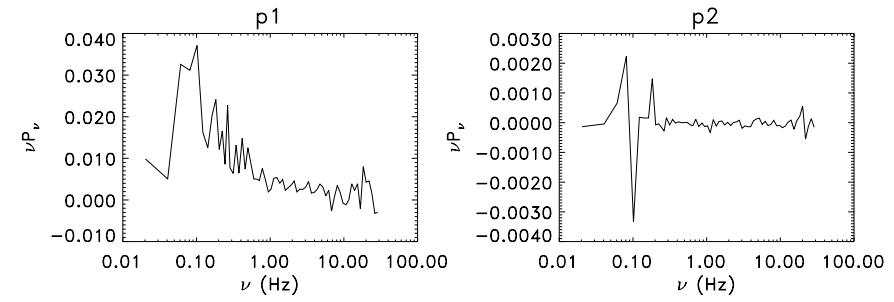

Fig. 10. Principal components of the variability in the X-ray power spectra. (Note that the vertical scale is linear, instead of the log scale in Fig. 9.)

The variation of the power spectrum can be studied with dynamical power spectra; such studies have been reported for many sources (cf. van der Klis 2000). In the spirit of our analysis of the cross correlation, the variability in the power spectra can also by studied by PCA. Treating the 297 individual power spectra in the same way as the cross correlations yields components shown in Fig. 10.

The first component of the X-ray power spectrum is rather similar to the average, with a broad peak around $0.1 \mathrm{~Hz}$. The second component shows a dip at $0.1 \mathrm{~Hz}$ and peaks at $0.08 \mathrm{~Hz}$ and $0.2 \mathrm{~Hz}$. This could be explained by the presence of QPOs at frequencies of $0.08,0.1$ and $0.2 \mathrm{~Hz}$, with the amplitude of the $0.1 \mathrm{~Hz}$ peak decreasing when the others are increasing. Tests with synthetic data show, however, that PCA also produces such a pattern if there is just a single a peak at a somewhat variable frequency. This seems a more likely interpretation in view of what is known about QPOs in similar sources.

The optical power spectra are affected by telescope vibrations at frequencies near 10 and $40 \mathrm{~Hz}$. As explained above, these vibrations were caused by the rather strong wind loading of the telescope on several of the nights. The optical variability is also contaminated with seeing effects, which have frequencies in the range range of interest here. Since the $\mathrm{X}-\mathrm{O}$ cross correlation reaches maximum values around $40 \%$ it is unlikely that seeing effects dominate the power spectrum, but its precise shape can not be reliably deduced from our observations.

With this caveat, the optical power spectrum shown in Fig. 9 is much broader than the X-ray power spectrum, and there is no clear evidence for the QPO seen in the X-rays. It thus seems unlikely that a QPO contributes strongly to the $\mathrm{X}-\mathrm{O}$ cross correlations. The effect of the X-ray QPO's on our cross correlations may well be just a a random noise contribution. 


\section{Conclusions and discussion}

We have studied the correlation of X-ray and optical properties of the black hole transient KV UMa. Thanks to the length of the dataset, the statistical properties of this correlation could be studied in detail. By analysis of the time series themselves, we find that the optical response to an X-ray peak consists of a broad dip followed by a sharper peak. By cross-correlating the two time series the same pattern is found, but with a much higher signal-to-noise ratio. From the cross correlation we find that the onset of the peaks in the optical response coincides with the X-ray maximum to within $30 \mathrm{~ms}$, and their initial rise is very fast. The minimum of the dip in the optical precedes the $\mathrm{X}$-rays by about $2 \mathrm{~s}$.

The agreement of the pattern of the cross-correlation with that found directly in the time series establishes, on the one hand, that the dip and the delayed peak are both properties of the optical light curve rather than the X-rays, and on the other hand that they are not due to artefacts of background subtraction or the cross-correlation process.

The shape of the cross correlation was found to be highly variable on time scales from minutes to days. A principal component analysis (PCA) of this variablity was found to be of some value in studying its statistical properties. It turned out that the principal components themselves, except for the first, are not very useful since their shape is highly affected by artefacts arising from a strong, randomly variable additive noise component in the cross correlation.

We find that the PCA components can be used, however, as an intermediate representation of the data, which can then be interpreted by a forward modeling process. The result of such a simulation shows that there are two systematic components which vary statistically independently. Both have the shape of a broad dip followed by a sharper peak, but their duration differs by a factor of about 3 .

The most puzzling aspect of the optical variations is of course the "precognition dips". If the optical light is due to reprocessing of X-rays, then an optical signal preceding the $\mathrm{X}$-rays would have to be due to something in the accretion flow preceding an X-ray event. In principle, this could happen if the increase in mass flux which somewhat later is observed as an increase of the X-ray flux, starts at some distance from the hole, such that the accretion time scale from that distance is of the order of a few seconds. For typical accretion disk models, this would be a distance intermediate between the X-ray emitting region and the optical reprocessing regions in the outer disk. If this event is associated with a local thickening of the accretion disk, and the X-ray emitting region is small enough, such a "bump" on the disk surface might be sufficient to shield the outer disk temporarily from the X-rays, leading to a dip in the optical emission.

While this possibility may be attractive in the reprocessing model, reprocessing itself has difficulties explaining the shape of the optical response. The sudden change in slope at zero lag turns out to be impossible to reproduce with any positive definite disk reprocessing function. The main reason for this is that the sudden change in slope requires much more variability on very short time scales $(<100 \mathrm{~ms})$ than the X-ray light curve actually has. A second difficulty is that the optical-to-Xray flux ratio in KV UMa was quite high, or the order $10 \%$. If, as the spectral energy distribution of McClintock et al. suggests, the UV-EUV emission is part of the same process producing the visible light, the reprocessed flux would have to be as large as the X-rays themselves.

From these difficulties it appears that alternatives may have to be explored to explain the visible light in black hole accreters like KV UMa and GX 339-4. In a future paper we will explore the possibility that the visible light is produced as thermal cyclo-synchrotron emission in the inner $20000 \mathrm{~km}$ of the accretion disk, as first suggested by Fabian et al. (1982) for GX 339-4 (see also Merloni et al. 2001 for an interpretation of the spectrum of KV UMa). If this interpretation is correct, it suggests a new and potentially very powerful means of probing the inner accretion flow by means of optical, and especiallly coordinated X-ray and optical observations.

Acknowledgements. We thank the XTE time allocation team and Skinakas observatory for their support and flexibility in making these observations possible, Dr. C. Straubmeier and F. Schrey, who carried out the optical observations and Dr. T. Belloni, who provided the XTE count rate data. We thank Dr. Christian Motch for his comments on an earlier version of this text. This work was done with support from the European Commission under grant no ERB-FMRX-CT98-0195.

\section{References}

Arons, J., \& King, I. R. 1993, ApJ, 413, L121

Boroson, T. A. 2002, ApJ, 565, 78

Brunt, C. M., \& Kerton, C. R. 2002, ApJ, 567, L41

Fabian, A. C., Guilbert, P. W., Motch, C., et al. 1982, A\&A, 111, L9

Folkes, S., Ronen, S., Price, I., et al. 1999, MNRAS, 308, 459

Haswell, C. A., Skillman, D., Patterson, J., Hynes, R. I., \& Cui, W. 2000, IAU Circ., 7427

Hynes, R. I., O’Brien, K., Horne, K., Chen, W., \& Haswell, C. A. 1998, MNRAS, 299, L37

Hynes, R. I., Mauche, C. W., Haswell, C. A., et al. 2000, ApJ, 539, L37

Kanbach, G., Straubmeier, C., Spruit, H. C., \& Belloni, T. 2001, Nature, 414, 180

Kanbur, S. M., Iono, D., Tanvir, N. R., \& Hendry, M. A. 2002, MNRAS, 329, 126

Kendall, M. S. 1980, Multivariate analysis, 2nd edition (Griffin, London)

Kong, A. K. H., Homer, L., Kuulkers, E., Charles, P. A., \& Smale, A. P. 2000, MNRAS, 311, 405

Matsuoka, M., Mitsuda, K., Ohashi, T., et al. 1984, ApJ, 283, 774

McClintock, J. E., Garcia, M. R., Caldwell, N., et al. 2000, ApJ, 551, L14

McClintock, J. E., Haswell, C. A., \& Garcia, M. R., et al. 2000, ApJ, 555,477

Merloni, A., Di Matteo, T., \& Fabian, A. C. 2001, ApSSS, 276, 213

Motch, C., Ilovaisky, S. A., \& Chevalier, C. 1981, IAU Circ., 3609

Motch, C., Ricketts, M. J., Page, C. G., Ilovaisky, S. A., \& Chevalier, C. 1983, A\&A, 119, 171 
O’Brien, K., \& Horne, K. 2000, in Rossi2000: Astrophysics with the Rossi X-ray Timing Explorer, NASA Goddard Space Flight Center, Greenbelt, MD USA, E91

Pavlenko, E. P., Dmitrienko, E. S., Shakhovskoi, N. M., et al. 2001, ApSSS, 276, 63.

Remillard, R. A., Morgan, E., Smith, D., \& Smith, E. 2000, IAU Circ., 7389

Revnivtsev, M., Sunyaev, R., \& Borozdin, K. 2000, A\&A, 361, L37

Straubmeier, C., Kanbach, G., \& Schrey, F. 2001, Exp. Astron., 11, 157
Turner, M. J. L., Breedon, L. M., Ohashi, T., Courvoisier, T., \& Inoue, H. 1985, SSRv, 40, 249

van der Klis, M. 2000, ARA\&A, 38, 717

van Paradijs, J., van der Klis, M., van Amerongen, S., et al. 1990, A\&A, 234, 181

Wagner, R. M., Foltz, C. B., Shahbaz, T., Casares, J., et al. 2001, ApJ, 556,42

Wood, K. S., Ray, P. S., Bandyopadhyay, R. M., et al. 2000, ApJ, 544, L45 